RECyT

Año 22 / № 33 / 2020 / 108-115

\title{
Sistema electrónico experimental para la medición de variables de parámetros de marcha en ancianos
}

\section{Experimental electronic system for the measurement of gait parameter variables in elderly people}

\author{
Jaime Menéndez Álvarez ${ }^{1, *}$, Gianna Arencibia Castellanos ${ }^{1}$, \\ Fidel E. Hernández Montero' ${ }^{1}$, Juan R. Rodríguez Suárez² ${ }^{2}$ Ailén Pérez Molinet ${ }^{1}$ \\ 1- Universidad Tecnológica de La Habana "José Antonio Echeverría" (CUJAE), Cuba \\ 2- Universidad de Pinar del Río, Cuba \\ *E-mail: jaimemenendezalvarez@gmail.com \\ Recibido el 10 de diciembre de 2019, Aprobado el 28 de abril de 2020.
}

\section{Resumen}

En el estudio actual que se realiza en Cuba sobre el monitoreo de indicadores del desempeño físico en ancianos, los parámetros medidos son afectados por la subjetividad del especialista que realiza las mediciones y no se tiene en cuenta la variabilidad que puede existir en estos. Desarrollar y evaluar en un entorno de laboratorio un sistema electrónico, de bajo costo, capaz de medir de forma inalámbrica estas variables físicas brinda la posibilidad de obtener parámetros de la marcha de manera automática. El sistema diseñado está basado en una Unidad de Medición Inercial (IMU) por tanto es capaz de medir la aceleración lineal y la velocidad angular en los tres ejes coordenados, así como la temperatura. El sistema transmite los datos de forma inalámbrica vía Bluetooth hacia una PC donde se implementa la etapa de procesamiento. Se evalúa el sistema de medición diseñado con diferentes pruebas que demuestran un comportamiento satisfactorio. También se muestran los resultados obtenidos al usar el dispositivo, en una prueba de equilibrio dinámico; en la cual las variables físicas obtenidas del IMU son procesadas con el fin de obtener el ángulo de inclinación del sensor.

Palabras clave: IMU; Sistema de Adquisición; Arduino; Indicadores de desempeño físico.

\section{Abstract}

This research, which is carried out in Cuba, is about the monitoring of physical performance indicators on elderly people. The parameters under measurement are affected by the specialist subjectivity who measures the parameters without carefully considering the variability that may exist among them. Developing and evaluating, within a laboratory environment, a low-cost electronic system capable of wirelessly measuring these physical variables, allows the possibility to automatically obtain gear parameters. This system is based on an Inertial Measurement Unit (IMU) that is able to measure not only the linear acceleration and angular speed in the three coordinate axes but also the temperature. At the same time, the system transmits data, via Bluetooth, to a PC where signal processing techniques are implemented. The designed measurement system is evaluated through different proofs which demonstrate a satisfying behavior. The obtained results are also shown when using this system in a dynamic equilibrium test in which the physical variables obtained by the IMU are processed to get the sensor angle inclination.

Keywords: IMU; Acquisition System; Arduino; Physical performance indicators.

\section{Introducción}

El envejecimiento es un fenómeno irreversible, común a todas las especies (Guerra et al., 2018). Estudios demográficos recientes estiman que la población actual de más de 65 años se duplicará en el año 2030, por tanto el cuidado y monitoreo de la salud del adulto mayor se hace cada vez más necesario (Rodríguez et al., 2017). Los indicadores de Desempeño Físico reflejan la capacidad para realizar movimientos o actividades específicas tales como: Velocidad de la Marcha, Fuerza de Agarre y Equilibrio. Estos contribuyen a medir el grado de funcionalidad en los ancianos y entre los indicadores más utilizados está la marcha, sus parámetros se muestran en la Tabla 1 ( Best et al., 2017, Jerome et al., 2015, Aboutorabi et al., 2016). En Cuba se han realizado estudios sobre los indicadores del desempeño físico en ancianos (Agustín \& Capote, 2010, Agustín et al., 2013, Agustín et al., 2018). Esto ha permitido la correcta caracterización de los mismos y la identificación de adultos mayores frágiles. Los procedimientos para medir estos in- 
dicadores se realizan mediante inspección visual por tanto, los resultados pueden verse afectados por la subjetividad del especialista que realiza las mediciones.

Para mejorar la calidad de estas pruebas y obtener mediciones más objetivas de los indicadores, es necesario automatizar las pruebas de desempeño físico. En la actualidad existen equipos basados en sensores inerciales que pueden ser usados para medir estos indicadores, es el caso del modelo MTi-10-series del fabricante Xsens el cual está formado por: IMU (Unidad de Medición Inercial), VRU (Unidad de Recuperación de Vapor) y AHRS (Sistema de Referencia de Actitud y Rumbo), con un costo de 800 euros. También del mismo fabricante existe el modelo MTi-100 basado en INS (Sistema de Navegación Inercial), IMU, VRU y AHRS, este provee datos con gran precisión como son: la posición, velocidad, aceleración y orientación, con un costo de 1850 euros. Ambos sensores vienen acompañados por un software que permite graficar los datos obtenidos y la comunicación entre el sensor y la computadora se hace mediante la interfaz RS232.

En este trabajo se desarrolla y evalúa un sistema electrónico que permite la medición inalámbrica de variables físicas necesarias para el monitoreo de la marcha humana, con un costo reducido en relación con desarrollos similares.

Tabla 1: Parámetros de la marcha.

\begin{tabular}{|c|}
\hline Cantidad de pasos \\
\hline Longitud del paso \\
\hline Cadencia (Cantidad de pasos/Tiempo) \\
\hline Velocidad \\
\hline Duración del paso \\
\hline Contacto inicial (inicio del paso) \\
\hline Contacto final(fin del paso) \\
\hline
\end{tabular}

\section{Materiales y Métodos}

El diseño del sistema se ha realizado en base a los siguientes requerimientos:

- Dispositivo capaz de registrar señales inerciales con una frecuencia de muestreo estable de hasta $200 \mathrm{~Hz}$.

- Posibilidad de almacenamiento de la información en una tarjeta micro SD.

- Transmisión de datos en tiempo real de manera inalámbrica empleando el protocolo Bluetooth.

- Visualización de datos en tiempo real en un ordenador.

- Interfaz sencilla, con posibilidad de definir parámetros como frecuencia de muestreo, modo de operación, rango de operación y el almacenamiento de los datos a un archivo.

- Sensor pequeño y portable.

Para las aplicaciones de monitorización de movimiento humano y de acuerdo con los requerimientos de usabilidad se requiere que el sensor sea pequeño y portable. El dispositivo debe poseer un acelerómetro y un giroscopio triaxial de bajo consumo y alta precisión. La frecuencia de muestreo aconsejable para poder medir el movimiento humano es de $40 \mathrm{~Hz}$. Sin embargo, es apropiado que el IMU tenga una frecuencia de muestreo de hasta $200 \mathrm{~Hz}$, ajustable por el usuario, abriendo la posibilidad de mejorar la precisión y de ampliar el abanico de aplicaciones para el dispositivo (Ribeiro \& Santos, 2017).

Los módulos de transmisión y almacenamiento de datos deben tener la posibilidad de inhabilitarse en función del modo de operación del sistema, ya sea para un comportamiento "online" u "offline". Se indica un comportamiento "online" a la transmisión continua de todos los datos obtenidos hacia una PC, vía Bluetooth, y su captura en tiempo real por una aplicación desarrollada en el ordenador. Un comportamiento "offline" solo incluiría la captura de datos y su almacenamiento en una tarjeta micro SD.

A continuación, se describe la arquitectura en bloques del hardware diseñado, así como los sensores inerciales incluidos. Se detallan las características del firmware desarrollado y el funcionamiento del programa.

\section{Hardware Desarrollado}

En la Figura 1: Diagrama en bloques del sistema de adquisición de datos. 1 se muestra un diagrama de bloques del diseño propuesto.

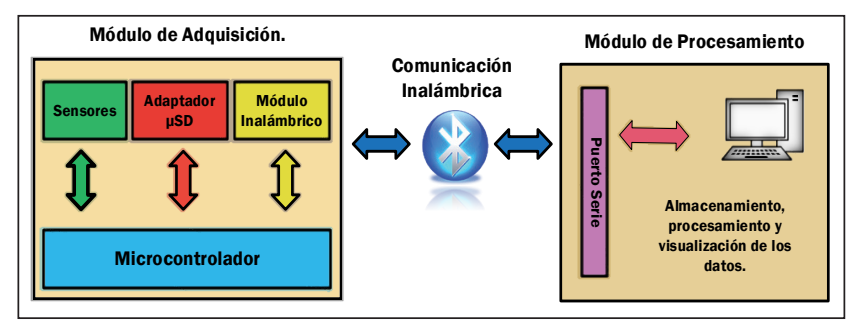

Figura 1: Diagrama en bloques del sistema de adquisición de datos.

El módulo de adquisición es el bloque que contiene los sensores inerciales y que envía los datos a una PC para su procesamiento y visualización, o bien almacenarlos en una tarjeta micro SD para su análisis posterior. Este módulo es el corazón del sistema y está formado por un Arduino Nano, la unidad de medición inercial MPU-6050, un módulo Bluetooth utilizado para la transmisión inalámbrica de datos, un adaptador micro SD para el almacenamiento y una batería de $9 \mathrm{~V}$.

El Arduino Nano es una tarjeta pequeña, perteneciente a la gran familia Arduino, ideal para proyectos donde el tamaño es una limitante. El Arduino está controlado por el ATMEGA328P, un microcontrolador de bajo consumo de 8 bits de Atmel basado en RISC. La tensión de funcionamiento del dispositivo se encuentra entre 1.8-5.5V.

El MPU-6050 es el sensor seleccionado para el diseño debido a su precisión, bajo costo y amplio uso en la literatura para el estudio de la marcha (Kodappully et al., 2017, Dinh et al., 2017, Ibarra et al., 2018, Xu et al., 2016, Kardos et al., 2017, Wang et al., 2015, Shiao et al. 2017). 
Posee un acelerómetro y un giroscopio en los tres ejes con una alta precisión, así como un sensor de temperatura.

De acuerdo con los requerimientos, el dispositivo se debe comunicar inalámbricamente con el ordenador para proporcionar los datos de los sensores en tiempo real mediante Bluetooth. El módulo Bluetooth seleccionado para el diseño es el HC-05, ya que es compatible con la mayoría de los dispositivos por implementar Bluetooth 2.0, es de bajo costo y es ampliamente utilizado en la literatura para el desarrollo de sistemas utilizando microcontroladores (Kodappully et al., 2017, Dinh et al., 2017, Kardos et al., 2017, Caliskan et al., 2018, Ma et al., 2018, Lee et al., 2018, Mazumder \& Vashista, 2017, Shandilya et al., 2015, Mathur et al., 2016). Ha sido configurado a una velocidad de transferencia de 115200 baudios en modo esclavo y posee un consumo de $31.5 \mathrm{~mA}$ de acuerdo con sus especificaciones. Es un dispositivo clase 2 por lo que permite un rango de alcance de unos $10 \mathrm{~m}$, pudiendo ser mayor en espacios cerrados por la presencia de reflexiones. La tensión de alimentación es de desde 3.5 hasta $6 \mathrm{~V}$, ya que posee un regulador de $3.3 \mathrm{~V}$ integrado.

Dado que el dispositivo debe comportarse también como un sistema de almacenamiento de datos, es requerido que incluya una unidad de almacenamiento. Para ello, se incorpora un conector para tarjeta micro SD. La tensión de alimentación de los módulos es de $3.3 \mathrm{~V}$, pero en la mayoría de los módulos se incorpora un regulador que permite alimentarlo directamente a $5 \mathrm{~V}$.

El sistema está alimentado por una batería de $9 \mathrm{~V}$ y 1000 mAh. La autonomía se calcula de 20 horas aproximadamente cuando las condiciones son las siguientes: el sistema trabaja a una frecuencia de muestreo de $200 \mathrm{~Hz}$ mientras se almacenan los datos continuamente en la tarjeta micro $\mathrm{SD}$ de todos los sensores. Es posible enviar los datos en tiempo real a través del módulo Bluetooth a la frecuencia de muestreo seleccionada, pero esto hace que el rendimiento del dispositivo disminuya. Cuando se envían los datos a 200 $\mathrm{Hz}$, vía Bluetooth, se estima que la autonomía decrece de 20 horas a 10 horas, ya que el consumo de dicho módulo es más alto que el consumo exigido por la tarjeta micro SD en el proceso de almacenaje de datos.

El dispositivo diseñado posee dos modos principales de operación: "online" y "offline". Si se selecciona el modo "online", el microcontrolador leerá los datos del sensor y los transmitirá de manera continua vía Bluetooth hacia una PC. La aplicación es capaz de graficar las siete señales medidas por el sensor, que incluyen la aceleración en los tres ejes, la velocidad angular en los tres ejes y la temperatura del dispositivo en grados Celsius. En el modo de operación "offline", los datos serán continuamente almacenados en una tarjeta micro SD conectada al módulo de adquisición para su posterior análisis, permitiendo la utilización del dispositivo sin restricciones de distancias y elevando la autonomía del dispositivo.

Las conexiones del módulo de adquisición de datos se muestran en las Figura 3: Diagrama en Bloques del
Módulo de Adquisición. Firmware Desarrollados 2 y 3.

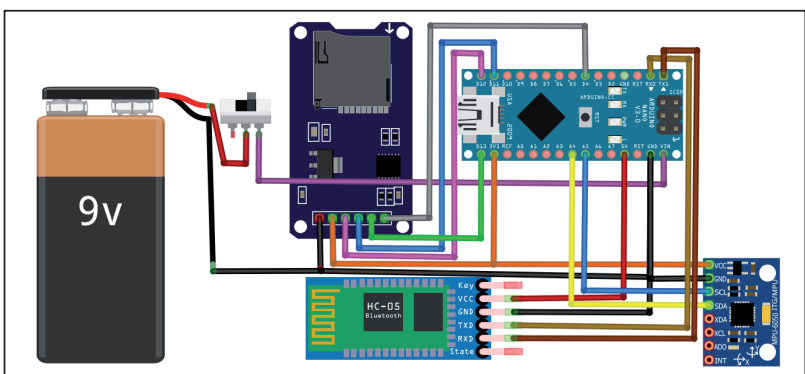

Figura 2: Diagramas de conexiones del Módulo de Adquisición.

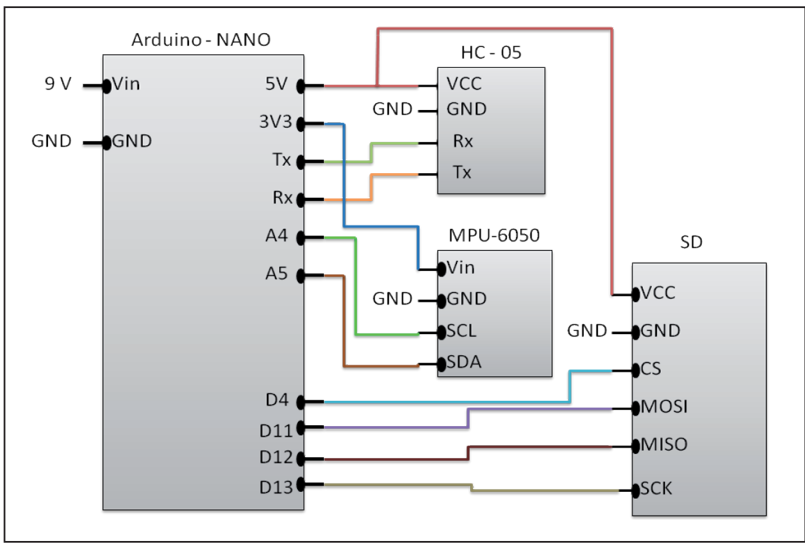

Figura 3: Diagrama en Bloques del Módulo de Adquisición. Firmware Desarrollado

El firmware es el encargado de gobernar los periféricos que intervienen en el sistema. La principal prioridad del dispositivo es garantizar una frecuencia de muestreo estable y precisa. El módulo de Bluetooth realiza dos funciones principales en el dispositivo. La primera es recibir instrucciones de configuración del ordenador para establecer los parámetros del dispositivo. La segunda es enviar los paquetes de datos en tiempo real con el fin de que se puedan procesar o almacenar en un sistema externo. En la Tabla 2 se muestran los modos de operación del dispositivo. La tarjeta micro SD que se empleó en el dispositivo tiene una capacidad de 8 GB y funciona a través de un sistema de archivos FAT32. Puesto que las tarjetas micro SD son dispositivos de memoria flash, los procesos para escribir bloques de datos a través de la SPI hacia la tarjeta SD pueden tardar más de 250 ms.

Tabla 2: Modos de operación del dispositivo.

\begin{tabular}{|c|c|}
\hline Modo de operación & Acción \\
\hline 0 & $\begin{array}{c}\text { Establece en el dispositivo el modo "offline" } \\
\text { e inicia el proceso de captura almacenando } \\
\text { los datos en la memoria micro SD. }\end{array}$ \\
\hline 1 & $\begin{array}{c}\text { Establece en el dispositivo el modo "online" } \\
\text { e inicia el proceso de captura transmitiendo } \\
\text { continuamente todos los datos mediante Bluetooth. }\end{array}$ \\
\hline 2 & $\begin{array}{c}\text { Detiene el proceso de captura de datos y pone al } \\
\text { dispositivo en espera de una nueva configuración. }\end{array}$ \\
\hline
\end{tabular}

Para hacer más eficiente la escritura en micro SD y evitar latencias innecesarias, la escritura en esta se realiza cuando hay 512 bytes de datos listos para escribir, es decir, cuando se han capturado 32 muestras de cada sensor. 


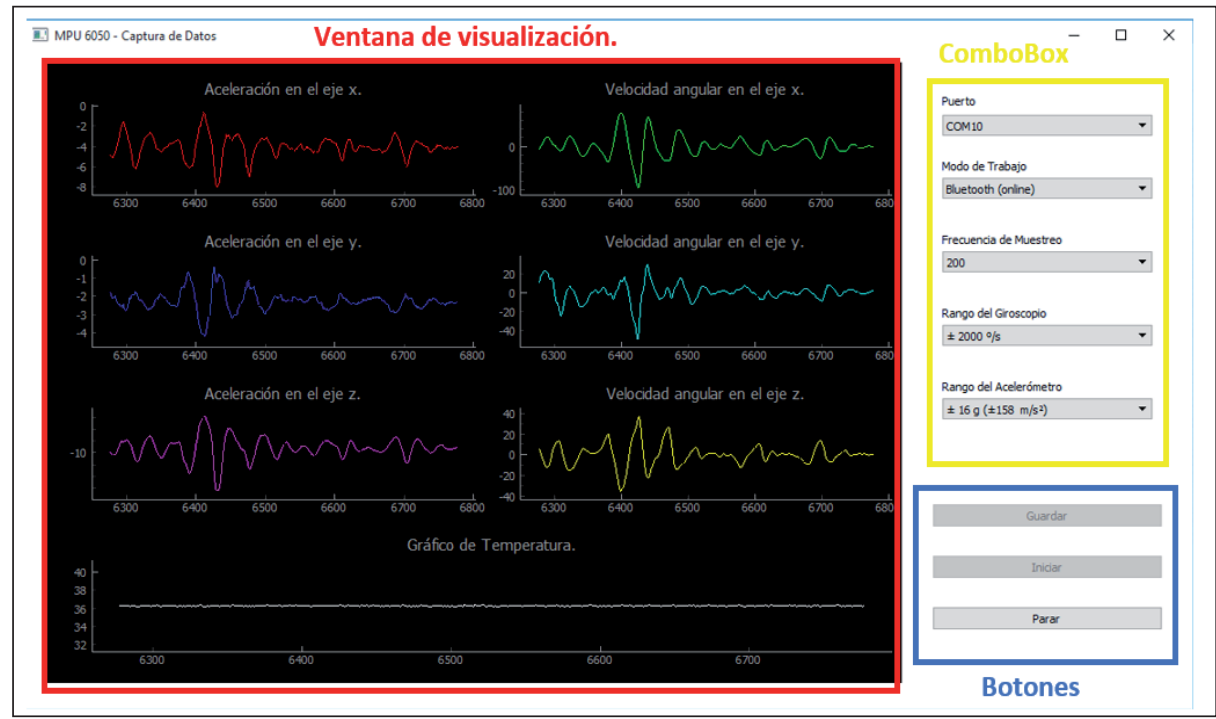

Figura 4: Interfaz gráfica de la aplicación.

Para el desarrollo de la aplicación en el ordenador se utilizó el lenguaje de programación Python en su versión 3.5 y como ambiente de desarrollo o IDE (Integrated Development Environment), el Spyder. Los módulos utilizados en Python para la implementación del programa son: Numpy, paquete que provee a Python con arreglos multidimensionales de alta eficiencia y diseñados para cálculo científico. PyQt5 y PyQtgraph, módulos utilizados para graficar en tiempo real la señal, así como para implementar la interfaz gráfica de la aplicación. PySerial, módulo utilizado para el manejo del puerto serial. Se utilizó la librería PyQt5 para el desarrollo de la interfaz gráfica. La aplicación tiene una interfaz conformada por una ventana de visualización, en la que se pueden observar los datos en tiempo real adquiridos por el sensor. La interfaz gráfica se muestra en la Figura 4: Interfaz gráfica de la aplicación.4.

$\mathrm{Al}$ abrir el programa, se realiza una búsqueda de todos los puertos seriales disponibles en el PC, por lo que es fundamental conectar el módulo de adquisición, o emparejarlo vía Bluetooth con el ordenador antes de iniciar la aplicación y seleccionar el puerto correcto. Al seleccionar el puerto, la frecuencia de muestreo, los rangos del sensor, el modo de trabajo y luego presionar el botón de inicio, automáticamente la aplicación abrirá el puerto seleccionado y enviará un paquete de configuración al módulo de adquisición vía Bluetooth con los parámetros seleccionados en la interfaz de usuario. Al detener la adquisición de datos con el botón de Parar, se envía al módulo de adquisición un paquete de configuración con la orden de detener estableciendo el campo modo de operación seleccionado. Se ofrece además la opción de guardar los datos recopilados en la ubicación y con el nombre deseado.

Cada paquete de datos recibido por el programa es almacenado en el buffer de entrada del puerto serie. Cada 50 ms todos los datos en el buffer de entrada del puerto serial es procesado, agregando los nuevos valores a las gráficas y guardando los datos en un buffer de datos. Luego, cada 10 segundos, todos los valores disponibles en el buffer de datos son salvados a un archivo temporal y el buffer se vacía.

\section{Resultados y Discusión}

Fue construido un prototipo de prueba con la finalidad de evaluar su funcionamiento y desempeño usable en las investigaciones que sobre desempeño físico en ancianos se llevan a cabo. Las evaluaciones del sistema se realizaron sobre el proceso de construcción en diferentes fases, y luego fueron integrados cada uno de los resultados al unificar los módulos. En la Tabla 3: Costo del prototipo desarrollado. 5 se muestra el prototipo diseñado sin el adaptador micro SD y en la Tabla 3, el costo de sus componentes, para lo cual los precios de estos fueron consultados en el año 2019 en la página web de Digi-Key.

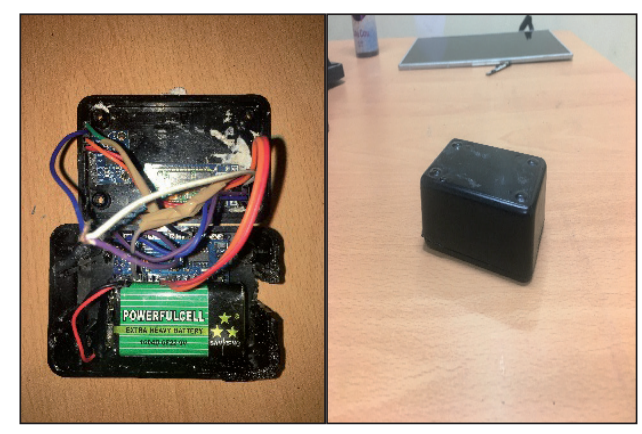

Figura 5: Prototipo del Sistema propuesto.

Tabla 3: Costo del prototipo desarrollado

\begin{tabular}{|c|c|c|}
\hline & Cantidad & Precio (USD) \\
\hline Arduino Nano - ATmega328P & 1 & 3.99 \\
\hline MPU-6050 & 1 & 2.80 \\
\hline Bluetooth HC-05 ZS-040 & 1 & 4.99 \\
\hline Batería de 9V & 1 & 7.99 \\
\hline Lector de Tarjetas micro SD Arduino & 1 & 1.55 \\
\hline Costo de los componentes & & 21.32 USD \\
\hline Pruebas técnicas & & 50 \\
\hline Honorarios por el trabajo de los diseñadores & & 100 \\
\hline Costo total & & 171.32 USD \\
\hline
\end{tabular}


La primera fase de pruebas inició con la puesta en marcha del sensor y la evaluación de la máxima frecuencia de muestreo del sistema. La frecuencia máxima de la Unidad de Medición Inercial MPU-6050 es de $1 \mathrm{kHz}$ para el acelerómetro, y $8 \mathrm{kHz}$ para el giroscopio.

El objetivo del experimento es comprobar la máxima frecuencia de muestreo a la cual la tarjeta Arduino Nano, trabajando a $16 \mathrm{MHz}$, es capaz de obtener los siete datos de 16 bits del sensor y transmitirlos vía serie hacia el ordenador. Para ello, fue utilizado cable mini USB para conectar el Arduino Nano con el ordenador. Para las pruebas se configuró el puerto serial del Arduino a 115200 baudios.

Se encontró que no era posible la transmisión de los datos a la velocidad de 115200 baudios si se utiliza una frecuencia de muestreo de $1 \mathrm{kHz}$. Cada paquete tiene un tamaño de 16 bytes y cada byte se transmite mediante el puerto serial acompañado de un bit de parada. Teniendo en cuenta esto, para cada paquete es necesario transmitir 16 bytes $* 9$ bits $=144$ bits. Si se divide la frecuencia del puerto serial 115200 baudios entre 144 bits se encontró que la máxima frecuencia de muestreo que se puede utilizar a 115200 baudios es 800 Hz. Para comprobarlo, se configuró una frecuencia de muestreo de $1 \mathrm{kHz}$ en el sensor, y se comprobó mediante análisis visual que la señal obtenida y graficada en el ordenador estaba completamente distorsionada y no respondía a los movimientos del sensor, como se muestra en la Figura 6: Señal distorsionada obtenida al establecer $1 \mathrm{kHz}$ de muestreo.6. Esto ocurre por el "cuello de botella" que se produce al muestrear tan rápido y no poder transmitir todos los datos obtenidos.

Se realizaron pruebas a diferentes frecuencias de mues- treo procediéndose de la siguiente manera: Se configuró la frecuencia de muestreo en el sensor y se habilitó un contador para la transmisión de 200000 muestras. En la PC, y a través del puerto serial se recibían estas muestras y se guardaban, además de graficarlas en tiempo real. La evaluación se realizó mediante inspección visual y el análisis de los datos obtenidos. A medida que se obtienen los datos en tiempo real, es posible apreciar el comportamiento de la señal con la observación de las gráficas en el programa diseñado. Si el sensor está en reposo, los ejes de las velocidades angulares deberán estar con valor nulo, y solo se medirá, según la posición del sensor, la aceleración de la gravedad de $9,8 \mathrm{~m} / \mathrm{s}^{2}$ en el eje perpendicular a la superficie terrestre. Además, mientras funciona el sensor, se puede apreciar de manera visual como responde a las vibraciones y movimientos del dispositivo. En el ordenador también se analiza la integridad de los datos. Para ello se verifica que el fichero guardado contenga las 200000 muestras que se transmiten con los 16 bytes cada uno.

Los resultados arrojaron que la máxima frecuencia a la que es posible trabajar con el dispositivo, configurando el puerto serial a 115200 baudios, es de $727.3 \mathrm{~Hz}$. A esta frecuencia se obtuvieron los 200000 datos transmitidos en el ordenador sin errores, además de que el análisis visual reflejó la correcta obtención de las muestras. Se realizaron los mismos experimentos a la velocidad de $666,6 \mathrm{~Hz}$ y 615.4 Hz con los mismos resultados.

Se probó la velocidad del puerto serial a 230400 baudios y se realizaron las pruebas descritas con frecuencias de muestreo de hasta un $1 \mathrm{kHz}$, sin distorsión en la señal y obteniéndose todos los datos transmitidos sin errores.

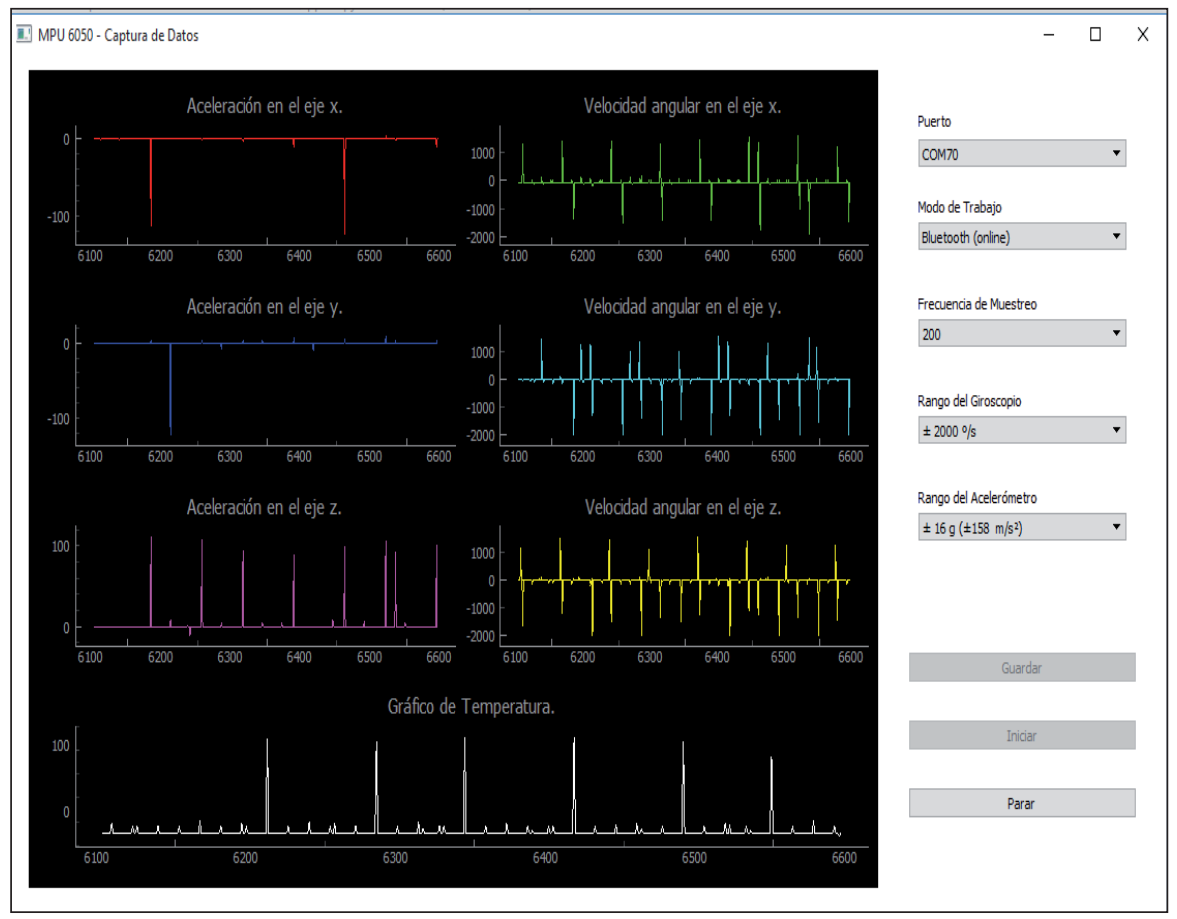

Figura 6: Señal distorsionada obtenida al establecer $1 \mathrm{kHz}$ de muestreo. 
Debido a que algunas versiones del módulo Bluetooth HC06 no soportan velocidades por encima de 115200 baudios, y a que algunos sistemas operativos pueden dar problemas con estas velocidades, se decidió establecer el baudrate del puerto serial a 115200 baudios. No es necesario para los fines del dispositivo tomar muestras a valores mayores que 115200 baudios, ya que la frecuencia recomendada para medir los movimientos del ser humano es de $40 \mathrm{~Hz}$ y el requisito de diseño propuesto es de $200 \mathrm{~Hz}$.

Se realizaron pruebas en cuatro entornos: dos al aire libre y dos en espacios cerrados, como se describe en la Tabla 4. Se utilizó una PC del fabricante DELL, modelo Inspiron 15, serie 3000 con adaptador Bluetooth modelo 1705 del fabricante Qualcomm Atheros. El módulo Bluetooth HC-05 fue configurado a una velocidad de transmisión de 115200 baudios con un bit de parada y ningún bit de paridad. El programa implementado en el Arduino configuró la frecuencia de muestreo a $200 \mathrm{~Hz}$.

Se procedió de la siguiente manera: a partir de $8 \mathrm{~m} \mathrm{y}$ aumentando progresivamente la distancia en intervalos de 0,5 $\mathrm{m}$ se ubicó el dispositivo, se intentó establecer la conexión y en caso de ser exitosa, se tomaron muestras durante 5 minutos configurando el sensor a una frecuencia de $200 \mathrm{~Hz}$. Las muestras fueron adquiridas por un programa desarrollado en Python, que comprobaba que el número de secuencia del paquete que se obtenía era el correcto y que no se perdieran paquetes. Los resultados de las pruebas se muestran en la Tabla 4, donde se detallan el porciento de paquetes perdidos en la comunicación. Se comprobó, mediante el análisis de los números de secuencias incluidos en cada uno de los paquetes de datos, el porciento de paquetes que se perdieron durante la comunicación, los resultados obtenidos son esperados ya que se está trabajando con dispositivos Bluetooth de clase 2.

Tabla 4: Porcentaje de paquetes perdidos a diferentes distancias.

\begin{tabular}{|c|c|c|c|c|c|c|c|}
\hline \multirow{2}{*}{ Entorno } & \multicolumn{7}{|c|}{ Distancia } \\
\cline { 2 - 8 } & $\mathbf{8 ~ \mathbf { ~ }}$ & $\mathbf{9 ~} \mathbf{~}$ & $\mathbf{1 0 ~} \mathbf{~}$ & $\mathbf{1 1} \mathbf{~ m}$ & $\mathbf{1 2 ~} \mathbf{~}$ & $\mathbf{1 3 ~} \mathbf{~}$ & $\mathbf{1 4 ~} \mathbf{~}$ \\
\hline Espacio Abierto. & 0 & 0 & 0 & 0 & $12 \%$ & $34 \%$ & $60 \%$ \\
\hline $\begin{array}{c}\text { Espacio Abierto } \\
\text { con obstáculos }\end{array}$ & 0 & 0 & 0 & $17 \%$ & $31 \%$ & $60 \%$ & - \\
\hline $\begin{array}{c}\text { Espacio cerrado } \\
\text { en la misma } \\
\text { habitación. }\end{array}$ & 0 & 0 & 0 & $7 \%$ & $25 \%$ & $63 \%$ & - \\
\hline $\begin{array}{c}\text { Espacio cerrado } \\
\text { en habitaciones } \\
\text { adyacentes. }\end{array}$ & 0 & 0 & $21 \%$ & $48 \%$ & $60 \%$ & - & - \\
\hline
\end{tabular}

Entre los requerimientos del diseño está la inclusión de un adaptador para tarjetas de memoria micro SD, ya que el dispositivo podría ser utilizado para el monitoreo prolongado de la actividad física en entornos abiertos. Debido a que la lectura y escritura en estos dispositivos traen demoras en los procesos del sistema, se decidió evaluar cuál era la máxima frecuencia de muestreo a la que se puede trabajar en el dispositivo con micro SD sin que se pierdan datos.

Para minimizar el tiempo de escritura en micro SD se decidió grabar los datos en bloques de 512 bytes. En un bloque se pueden guardar 32 paquetes de 16 bytes. Si la escritura se realizara cada vez que se guarda un paquete, el proceso sería 16 veces más lento, por lo tanto, la escritura en bloques es una buena práctica para optimizar la velocidad del sistema.

Utilizando la función de biblioteca de Arduino micros(), que mide el tiempo en microsegundos desde que se inició el sistema, se implementó un programa que transmite, mediante el puerto serial, el tiempo que demora la escritura en la tarjeta SD. Para el manejo de la tarjeta se utilizó la librería SD, incluida en el entorno de desarrollo de Arduino. Al escribir más de 512 bytes, automáticamente se escribirá el bloque en la tarjeta y se cargará el próximo bloque. Se configuró el sensor a una frecuencia de 200 Hz y se utilizó una tarjeta micro SD de 8 GB clase 4 del fabricante japonés ADATA con sistema de archivos FAT32.

Los resultados de las pruebas arrojaron demoras variables de hasta $221 \mathrm{~ms}$ en la tarjeta micro SD. Este resultado limita la frecuencia de muestreo del dispositivo al utilizar la tarjeta como modo de operación. Teniendo en cuenta que el buffer FIFO puede almacenar unos 32 valores de lectura de todos los sensores, la frecuencia máxima a la que se puede muestrear con esta latencia es de $144 \mathrm{~Hz}$. Por ello, el funcionamiento utilizando la tarjeta micro SD se ha limitado a 125 Hz como frecuencia máxima de trabajo (modo "offline").

\section{Aplicación Real del Sistema}

El sistema fue aplicado en las pruebas de evaluación de equilibrio clínico que se desarrollan en el Hospital "General Calixto García", en particular, en el Centro de Investigación sobre Longevidad, Envejecimiento y Salud. Una de estas pruebas es la de equilibrio dinámico, la cual consiste en contar cuántas veces un adulto mayor cambia desde la posición de sentado a parado en un tiempo de 12 s. Para esto, se colocó el sistema electrónico en la forma que se muestra en la Figura 7: Sujeto realizando pruebas de equilibrio con el sistema propuesto.7, con el objetivo de usar la estimación del ángulo de orientación del IMU, en el plano transversal del cuerpo humano. Para estimar este ángulo se utilizó el algoritmo de filtro complementario, el cual fusiona los datos del acelerómetro y del giroscopio con el propósito de obtener una medida más exacta de los ángulos de inclinación del sensor (Peña, 2013). Luego, se contarán las subidas consideradas como válidas. Una subida será considerada como válida cuando la señal de ángulo estimado supere un umbral, que en este caso se establece de manera empírica, con valor de 75, dentro de un intervalo de $12 \mathrm{~s}$. 


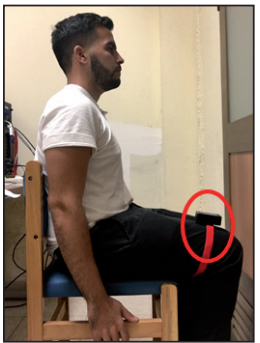

Figura 7: Sujeto realizando pruebas de equilibrio con el sistema propuesto.

Como resultado, y a modo de ejemplo, en la Se ha diseñado a través de un sistema basado en microcontrolador, con interfaz Bluetooth, una herramienta capaz de medir inalámbricamente las variables físicas necesarias para determinar parámetros correspondientes a indicadores de desempeño físico en ancianos. El dispositivo puede trabajar enviando los datos en tiempo real vía Bluetooth o bien guardando los datos en una tarjeta micro SD. Los datos recibidos inalámbricamente se grafican en tiempo real; y luego son procesados para determinar el ángulo de elevación que alcanzan los pacientes al realizar la prueba de equilibrio dinámico. 8 se muestra la estimación del ángulo obtenida al realizar una prueba, en la cual el cambio de posición se realiza de forma válida tres veces en $12 \mathrm{~s}$. Se aprecia que se opera de manera efectiva.

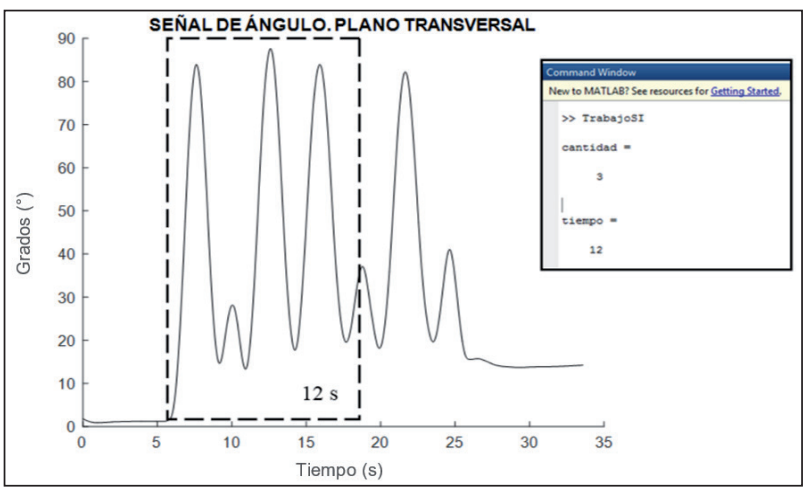

Figura 8: Señal de Ángulo, Plano Transversal.

\section{Conclusiones}

Se ha diseñado a través de un sistema basado en microcontrolador, con interfaz Bluetooth, una herramienta capaz de medir inalámbricamente las variables físicas necesarias para determinar parámetros correspondientes a indicadores de desempeño físico en ancianos. El dispositivo puede trabajar enviando los datos en tiempo real vía Bluetooth o bien guardando los datos en una tarjeta micro SD. Los datos recibidos inalámbricamente se grafican en tiempo real; y luego son procesados para determinar el ángulo de elevación que alcanzan los pacientes al realizar la prueba de equilibrio dinámico.

Se logró desarrollar un sistema electrónico a un costo más bajo que los sistemas que actualmente se comercializan, como son los casos del MTi-10 con un costo de 800 euros y el MTi-100 con un costo de 1850 euros. El perfeccionamiento de este sistema puede permitir en un futuro cercano realizar el diagnóstico y seguimiento de diversas enfermedades relacionadas con el deterioro cognitivo como por ejemplo la demencia. Así como la detección de ancianos frágiles y con riesgo de caídas. Esto se debe a que existe una estrecha relación entre los parámetros de la marcha y el estado cognitivo de una persona. En futuros trabajos también se propone comparar el sistema electrónico propuesto con otros que existen en el mercado en cuanto a precisión, robustez, confiabilidad, nivel de aplicación, entre otros aspectos, que nos permitan determinar la calidad de nuestro sistema así como su perfeccionamiento.

\section{Referencias}

1. Guerra, JJL; Marante, JPD; Salgado, AMR; Peñalver, A; Guerrero, EPG; Mola, E. R \& Palls, MT. E. (2018). Determinants of the health status of the population and its influence on the development of cognitive disorders. Revista Cubana de Salud Pública ; 44(1):141-152.

2. Rodríguez, JJ. L; Cepero, A. V; Medina, AM. L; Fernández, L. N; Álvarez R. P; Hernández, MA. G, et al. (2017). Cuba's aging and Alzheimer longitudinal study. MEDICC Rev. 2017;19:31-35.

3. Best JR, Ambrose T. L, Boudreau RM, Ayonayon HN, Satterfield S, Simonsick, EM, et al. (2016). An evaluation of the longitudinal, bidirectional associations between gait speed and cognition in older women and men. J Gerontol Ser Biomed Sci Med Sci. 2016;71(12):1616-1623.

4. Jerome, GJ; Ko, S; Kauffman, D; Studenski, SA; Ferrucci, L; Simonsick, EM. (2015) Gait characteristics associated with walking speed decline in older adults: results from the Baltimore Longitudinal Study of Aging. Arch Gerontol Geriatr. 2015;60(2):239-243.

5. Aboutorabi, A; Arazpour, M; Bahramizadeh, M; Hutchins, SW; Fadayevatan, R. (2016). The effect of aging on gait parameters in able-bodied older subjects: a literature review. Aging Clin Exp Res. 2016;28(3):393-405.

6. Agustín, DG; Capote, CB. (2010). Desempeño Físico en adultos mayores sanos, del municipio Plaza de la Revolución. GeroInfo. Vol. 5. N¹. 2010. ISSN 1816-8450.

7. Agustín, DG; García, JFP; de la Torre, JAP; Orihuela, MG; Osores, RM; González, AG. (2013). Comorbilidad y Desempeño en personas adultos mayores de La Habana, Cuba. Horiz Méd. 2013;13(2):19-27.

8. Agustín, DG; Morejón, SC; Pérez, ZR. (2018). Las pruebas de desempeño físico en el pronóstico de desenlaces adversos en los ancianos. MediSan. 2018;22(6):466-470.

9. Ribeiro, NF; Santos, CP. (2017). Inertial measurement units: A brief state of the art on gait analysis. En: IEEE 5th Portuguese Meeting on Bioengineering (ENBENG). 2017. p. 1-4.

10. Kodappully, M; Kumar, D; Lahiri, U. (2017). A step towards smart health: A pelvic wearable device for gait health 
quantification. En: 2017 IEEE Region 10 Symposium (TENSYMP). IEEE; 2017. p. 1-5.

11. Dinh, A; Bayati, M; Bhatti, M; Yepez, J; Zhexin, J. (2017). Design and implementation of a wireless wearable band for gait analysis. En: International Conference on the Development of Biomedical Engineering in Vietnam. Springer; 2017. p. 693-698.

12. Ibarra, JCP; Williams, H; Siqueira, AA; Krebs, HI. (2018). RealTime Identification of Impaired Gait Phases Using a Single Foot-Mounted Inertial Sensor: Review and Feasibility Study. En: 2018 7th IEEE International Conference on Biomedical Robotics and Biomechatronics (Biorob). IEEE; 2018. p. 1157-1162.

13. $\mathrm{Xu}, \mathrm{H} ; \mathrm{Gu}, \mathrm{L} ; \mathrm{Tu}, \mathbf{B}$. (2016). Gait authentication based on partial cyclic rotation metric. En: 2016 Chinese Control and Decision Conference (CCDC). IEEE; 2016. p. $1143-1148$.

14. Kardos, S; Balog, P; Slosarcik, S. (2017). Gait dynamics sensing using IMU sensor array system. Adv Electr Electron Eng. 2017;15(1):71-76.

15. Wang, B; Rajput, KS; Tam, W-K; Tung, AK; Yang, Z. (2015). FreeWalker: A smart insole for longitudinal gait analysis. En: 2015 37th Annual International Conference of the IEEE Engineering in Medicine and Biology Society (EMBC). IEEE; 2015. p. 3723-3726.

16. Shiao, Y; Chang, T-Y; Lai, C-H. (2017). Gait identification using inertial sensors for control of adaptive knee orthosis device. Sens Mater. 2017;29(11):1657-1665.

17. Caliskan, M; Tumer, AE; Sengul, SB. (2018). Development of a Prototype Using the Internet of Things for Kinetic Gait Analysis. Int J Intell Syst Appl Eng. 2018;6(3):242247.
18. Ma CZ. H; Zheng, Y. P; Lee WC. C. (2018). Changes in gait and plantar foot loading upon using vibrotactile wearable biofeedback system in patients with stroke. Top Stroke Rehabil. 2018;25(1):20-27.

19. Lee, C. N; Fong, V. H; Chu, Y. T; Cheng, L; Chuang, HW; Lo, C Y. (2018). A Wearable Device Of Gait Tracking For Parkinson'S Disease Patients. En: 2018 International Conference on Machine Learning and Cybernetics (ICMLC). IEEE; 2018. p. 462-466.

20. Mazumder, A; Vashista, V. (2017). Development of an Adaptive Gait Characterizer. En: Proceedings of the Advances in Robotics. ACM; 2017. p. 49.

21. Shandilya, S; Meena, MK; Kumar, N. (2015). A low cost wireless sensor development for assessing ground reaction force in gait analysis. En: 2015 Annual IEEE India Conference (INDICON). IEEE; 2015. p. 1-3.

22. Mathur, N; Glesk, I; Davidson, A; Paul, G; Banford, J; Irvine, J; et al. (2016). Wearable mobile sensor and communication platform for the in-situ monitoring of lower limb health in amputees. En: 2016 IEEE International Symposium on Circuits and Systems (ISCAS). IEEE; 2016. p. $1366-1369$.

23. Peña, R. v. (2013). Sistema de detección de movimientos basado en sensores inerciales integrados. Tesis para optar por el título de Maestro en Tecnología Avanzada, Instituto Politécnico Nacional, México. https://tesis. ipn.mx > Tesis Maestria Roberto Vela Peña. 